\title{
On the transparency of the universe for very high energy photons
}

\author{
Manuel Meyer* \\ Insitut für Experimentalphysik, University of Hamburg, Luruper Chaussee 149, 22761 Hamburg, \\ Germany \\ E-mail: manuel.meyer@physik.uni-hamburg.de
}

\section{Dieter Horns}

Insitut für Experimentalphysik, University of Hamburg, Luruper Chaussee 149, 22761 Hamburg,

Germany

E-mail: dieter.horns@physik.uni-hamburg.de

The transparency of the universe for very high energy (VHE, $E>100 \mathrm{GeV}$ ) photons from sources at cosmological distances is limited due to pair-production with low energy photons of the extra galactic background light (EBL) in the optical to infrared band. Here, we use all available energy spectra from VHE emitting active galactic nuclei to search for signatures of non-standard physics. A combined statistical study using a new approach shows evidence for a hardening of the intrinsic power laws at the transition from optical thin to optical thick regions. Additionally, we find a moderately significant $p$-value of the expected absorption effect.

Cosmic Radiation Fields: Sources in the early Universe - CRF2010,

November 9-12, 2010

Desy Germany

${ }^{*}$ Speaker. 


\section{Introduction}

The transparency of the universe for very high energy (VHE) photons with energies $E \gtrsim$ $100 \mathrm{GeV}$ is limited due to the interaction with background photon fields. The process $\gamma_{\mathrm{VHE}}+\gamma_{\mathrm{bkg}} \rightarrow$ $e^{+}+e^{-}$attenuates the intrinsic photon flux $f^{\text {deabs }}$ of a source at a redshift $z$ :

$$
f^{\mathrm{obs}}(E)=\exp \left[-\tau_{\gamma}(E, z)\right] f^{\text {deabs }}(E[1+z]),
$$

where $\tau_{\gamma}$ is called the optical depth. The optical depth is given by a threefold integral of the pairproduction cross-section and the background photon-field density over the distance of the source, the background photon field energy and the angle between the photon momenta [1]. The interaction rate peaks for a wavelength $\lambda_{*}$ of the background photons given by $\lambda_{*} \approx 1.4 \mu \mathrm{m}(E / \mathrm{TeV})$. Consequently, the diffuse extra galactic background light (EBL) in the UV to the far-IR regime is the most relevant photon field for the attenuation of $\mathrm{TeV}$ photons [2]. The EBL itself is given by the (redshifted) integrated starlight that has been emitted over all epochs and the absorbed and re-emitted light by dust in galaxies. Hence, one expects two maxima in the spectral energy distribution (SED) of the EBL. One at a wavelength of roughly $1 \mu \mathrm{m}$ (stellar) and the other around $100 \mu \mathrm{m}$ (dust). Direct measurements of the EBL are challenging given the dominating foreground emission. In order to calculate the pair opacity one has to rely on models that describe the SED of the EBL [3 -5]. If observed spectra are corrected using a certain EBL model they will be referred to as deabsorbed spectra with a flux $f^{\text {deabs }}$. The optical depth is further sensitive to effects induced by non-standard physics e.g. Lorentz invariance violation, as predicted by certain quantum gravity theories [6,7], or the presence of exotic particles that interact with the photons of cosmological sources during their propagation such as axions, axion like particles (ALPs) or hidden sector photons [8-11]. In the commonly discussed example of axions or ALPs photons could convert into these particles in intergalactic magnetic fields and vice versa.

The aim of this article is to introduce a new approach to test spectra of active galactic nuclei (AGN) for indications of new physics and to search for an EBL absorption feature. For this approach currently available energy spectra of AGN measured with the imaging air Cherenkov telescopes CAT, HEGRA, HESS, MAGIC, VERITAS and WHIPPLE are considered. Sources with unknown redshift are excluded from the test. The reference for each spectrum can be found elsewhere [12].

The new approach is based on the unbinned Kolmogorov-Smirnov test. It has the advantage that every individual data point of the spectra is included in the test. The only (testable) assumption that is needed is that the intrinsic or observed AGN spectra can be described by power laws (with an exponential cut-off if necessary). ${ }^{1}$ Neither any assumption about the spectral index is necessary nor does the test depend on the evolution of the spectral index with redshift [13, 14]. Moreover, the individual errors of the data points only enter in the fit of the power law to the data. The test itself is independent of the uncertainties of each observation.

\footnotetext{
${ }^{1}$ Henceforth, it will always be implied that either power laws or power laws with an exponential cut-off are fitted to the data where the latter case will not be written out explicitly in the following. Power laws with an exponential cut-off are used if the $p$-value of a simple power-law fit is below 0.05 .
} 


\section{Method: the Kolmogorov-Smirnov test to investigate VHE blazar spectra}

The new approach is based on the unbinned Kolmogorov-Smirnov (KS) test which is tests the null hypothesis $\mathrm{H}_{0}$ that two data sets with $N_{1}$ and $N_{2}$ data points are sampled from an identical underlying probability distribution function [15]. The data points are assumed to lie at values $x_{i}$, $i=1, \ldots, N_{1}$ and $\tilde{x}_{j}, j=1, \ldots, N_{2}$. Unbiased estimators of the probability distribution functions from which the measurements were drawn are given by the cumulative distribution functions (CDFs) $S_{N_{1}}(x)$ and $S_{N_{1}}(x)$. The measure if two sets of data points are drawn from the same underlying probability distribution is given by the maximum value $D$ of the difference between the two CDFs,

$$
D=\max _{-\infty<x<\infty}\left|S_{N_{1}}(x)-S_{N_{2}}(x)\right| .
$$

To quantify the discrepancy between the data and $\mathrm{H}_{0}$ the $p$-value is used which gives the probability to find a value of $D$ larger than the observed one under the assumption of $\mathrm{H}_{0}$. It is approximately given in terms of the function $Q_{\mathrm{KS}}$ by

$$
p \equiv \operatorname{Probability}(D>\text { observed })=Q_{\mathrm{KS}}\left(y=\left[\sqrt{N_{e}}+0.12+0.11 / \sqrt{N_{e}}\right] D\right),
$$

where

$$
N_{e}=\frac{N_{1} N_{2}}{N_{1}+N_{2}} \quad \text { and } \quad Q_{\mathrm{KS}}(y)=2 \sum_{j=1}^{\infty}(-1)^{j-1} \exp \left(-2 j^{2} y^{2}\right)
$$

Equipped with these formulae one is now able to search for deviations from standard physics and EBL absorption features.

\subsection{Search for exotic physics}

If particles such as axions or ALPs existed they would alter the optical depth especially for large values of $\tau_{\gamma}[6,11,10]$. There are only a few measurements available that correspond to optical depths beyond values of one since such large values translate to observations of distant sources $(z \gtrsim 0.2)$ at VHE energies beyond $\sim 1 \mathrm{TeV}$. As a compromise between expected deviations from standard physics and a reasonable number of data points to obtain a statistical significant result, a limiting value of $\tau_{\gamma}=2$ is chosen a priori above which effects induced by e.g. ALPs are searched for. Moreover, ALPs can reduce the photon flux maximally to $2 / 3$ of the initial value [16]. Such a drop is flux is also naturally expected because of the interaction of VHE photons with background photons at $\tau_{\gamma} \approx 0.41$. The value of $\tau_{\gamma}=2$ avoids any confusion between these two phenomena.

The null hypothesis $\left(\mathrm{H}_{0}\right)$ is that the intrinsic spectra follow power laws. The observed spectra are deabsorbed with a lower limit EBL model [4] and a power law is fitted to each spectrum but only to data points that correspond to an optical depth of $\tau_{\gamma}<1$. The fit is extrapolated to data points with a larger value of $\tau_{\gamma}$ and for the $i$-th measurement of these data points the ratio $R_{i}^{\text {deabs }}=\left(f_{i}^{\text {deabs }}-f_{i}^{\text {ext }}\right) /\left(f_{i}^{\text {deabs }}+f_{i}^{\text {ext }}\right)$ between the deabsorbed flux $f_{i}^{\text {deabs }}$ and the flux extrapolated from the fit $f_{i}^{\text {ext }}$ is calculated. If $\mathrm{H}_{0}$ is correct the following two distributions named $N_{\text {thin }}$ for optical thin and $N_{\text {thick }}$ for optical thick regions (i.e. $\tau_{\gamma} \geq 2$ ) should not differ significantly from each other,

$$
\begin{aligned}
N_{\text {thin }} & =\left\{R_{i}^{\text {deabs }} \mid 1 \leq \tau_{\gamma}\left(E_{i}, z\right)<2 ; \forall i\right\}, \\
N_{\text {thick }} & =\left\{R_{i}^{\text {deabs }} \mid \tau_{\gamma}\left(E_{i}, z\right) \geq 2 ; \forall i\right\},
\end{aligned}
$$


and should both scatter symmetrically around values of $R=0$. On the other hand, if the universe is more transparent to VHE photons for $\tau_{\gamma} \geq 2$ than predicted by the lower limit EBL model or the underlying assumptions of power laws foes not hold the ratios of $N_{\text {thick }}$ will increase since the applied correction is too large.

\subsection{Search for an EBL absorption feature}

The decrease in the SED of the EBL between the first maximum and the minimum (see Section 1) leads to a distinctive plateau between $1 \mathrm{TeV} \lesssim E \lesssim 5 \mathrm{TeV}$ at redshifts $z \lesssim 0.6$ in the optical depth [4] where $\tau_{\gamma}$ is almost constant in energy. Superimposed with a power law this leads to a bump in the observed spectra. ${ }^{2}$

To test whether this feature is indeed significant, power laws are fitted to the spectra this time excluding data points in the region between $1 \mathrm{TeV} \leq E \leq 5 \mathrm{TeV}$ from the fit. The bump should appear for observations that suffer from a high attenuation, thus the limit above which this absorption feature is expected to occur is set a priori to $\tau_{\gamma}=1$. The ratios are again defined as $R_{i}=\left(f_{i}^{\mathrm{obs}}-f_{i}^{\mathrm{ext}}\right) /\left(f_{i}^{\mathrm{obs}}+f_{i}^{\mathrm{ext}}\right)$.

The null hypothesis is formulated as follows: the distribution of the ratios for observed measurements for $\tau_{\gamma}<1$ is equal to the distributions of observed data points with $\tau_{\gamma} \geq 1$. One expects that below $\tau_{\gamma}=1$ the ratios scatter randomly around one whereas above $\tau_{\gamma}=1$ they should increase to values larger than one if the bump is present. This is again tested with the KS test by comparing the two distributions of ratios

$$
\begin{aligned}
N_{\text {nobump }} & =\left\{R_{i}^{\mathrm{obs}} \mid \tau_{\gamma}\left(E_{i}, z\right)<1 ; 1 \mathrm{TeV} \leq E_{i} \leq 5 \mathrm{TeV} ; \forall i\right\}, \\
N_{\text {bump }} & =\left\{R_{i}^{\mathrm{obs}} \mid \tau_{\gamma}\left(E_{i}, z\right) \geq 1 ; 1 \mathrm{TeV} \leq E_{i} \leq 5 \mathrm{TeV} ; \forall i\right\} .
\end{aligned}
$$

If the bump is significant and has its origin in the attenuation of VHE $\gamma$-rays by the EBL, deabsorbing the spectra and repeating the test with the deabsorbed ratios should lead to a decreased significance of this feature.

\section{Results}

\subsection{Search for exotic physics}

The result of the KS test is shown in Fig. 1. The upper panel shows the optical depth plotted against the ratios (blue diamonds for ratios of $N_{1}$ and red circles for ratios of $N_{2}$, see Eqns. 2.4 and 2.5). The lower panel shows the corresponding CDFs for the distributions $N_{\text {thin }}$ (blue line) and $N_{\text {thick }}$ (red line). The ratios with $1 \leq \tau_{\gamma}<2$ scatter around zero as expected under $\mathrm{H}_{0}$ as it is seen from CDF which is $\approx 0.5$ at $R=0$. On the other hand, the ratios of $N_{\text {thick }}$ show an systematic increase towards values larger than zero which indicates a hardening of the intrinsic spectra for $\tau_{\gamma} \geq 2$. The $p$-value for $\mathrm{H}_{0}$ is found to be $2.59 \times 10^{-3}$, hence there is evidence that the two distributions are different from each other. Albeit the large value of $D$ the significance of two different distributions does not exceed $\approx 3 \sigma$ due to the small number of ratios for $\tau_{\gamma} \geq 2$. If the significance of a disproof of $\mathrm{H}_{0}$ increases with future data either intrinsic AGN spectra are not well described by power laws or there might be an exotic mechanism at work that reduces the pair opacity for VHE photons.

\footnotetext{
${ }^{2}$ This feature is e.g. visible in the spectrum of 1ES 1101-232 [17]
} 


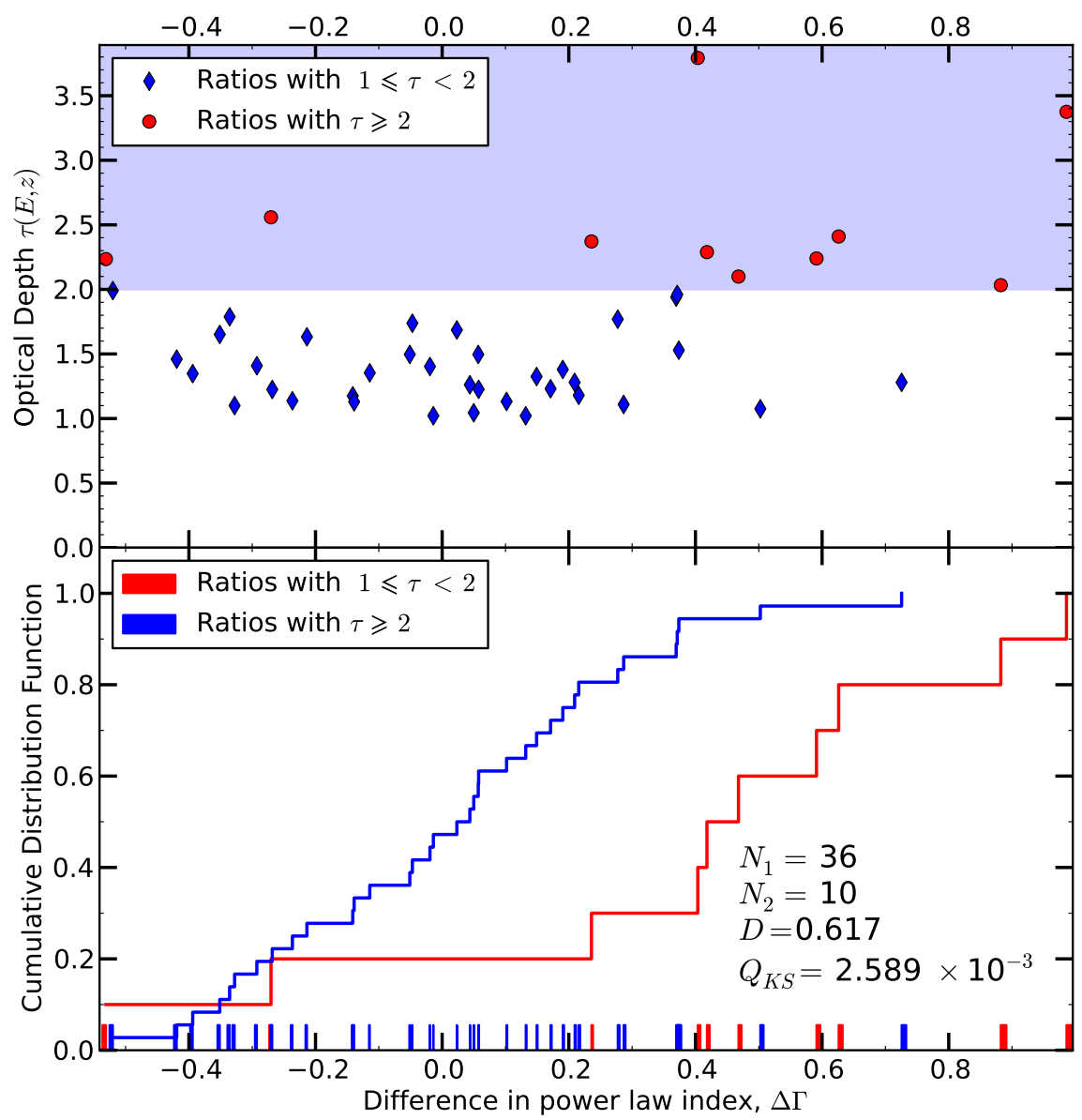

Figure 1: Result of the KS test searching for exotic physics. Upper panel: the optical depth is plotted against the ratios of the deabsorbed and the extrapolated flux. Above $\tau_{\gamma}=2$ (light blue shaded region) the ratios do increase but the difference is not significant. Lower panel: the CDFs for the two sets $N_{1}$ and $N_{2}$ of Eqn. 2.4 and 2.5. The maximum distance $D$ is 0.617 which results in a $p$-value of $2.589 \times 10^{-3}$ that the two distributions are equal and the intrinsic spectra follow power laws.

\subsection{Search for an EBL absorption feature}

Fig. 2 and 3 show the results of the KS test for the EBL absorption feature. Fig. 2 depicts the same as Fig. 1 but this time for the distributions defined in Eqn. 2.6 and 2.7. The majority of ratios with $\tau_{\gamma} \geq 1$ lies above $R=0$ which is apparent from the lower panel as the corresponding CDF shows a sharp increase at $R \approx 0.2$. On the other hand, the ratios with $\tau_{\gamma}<1$ do scatter around zero. The KS test results in a mildly significant probability that the distributions are different, i.e. the $p$-value is found to be $2.57 \times 10^{-2}$. Fig. 3 illustrates the result for the KS test for the deabsorbed spectra. The CDF for the ratios with $\tau_{\gamma} \geq 1$ now features a plateau between $R \approx 0$ and $R \approx 0.2$. As a consequence, the $p$-value that no bump is present is increased to $7.55 \times 10^{-2}$.

Hence, for the particular EBL model [4] considered here an absorption feature is found with a 


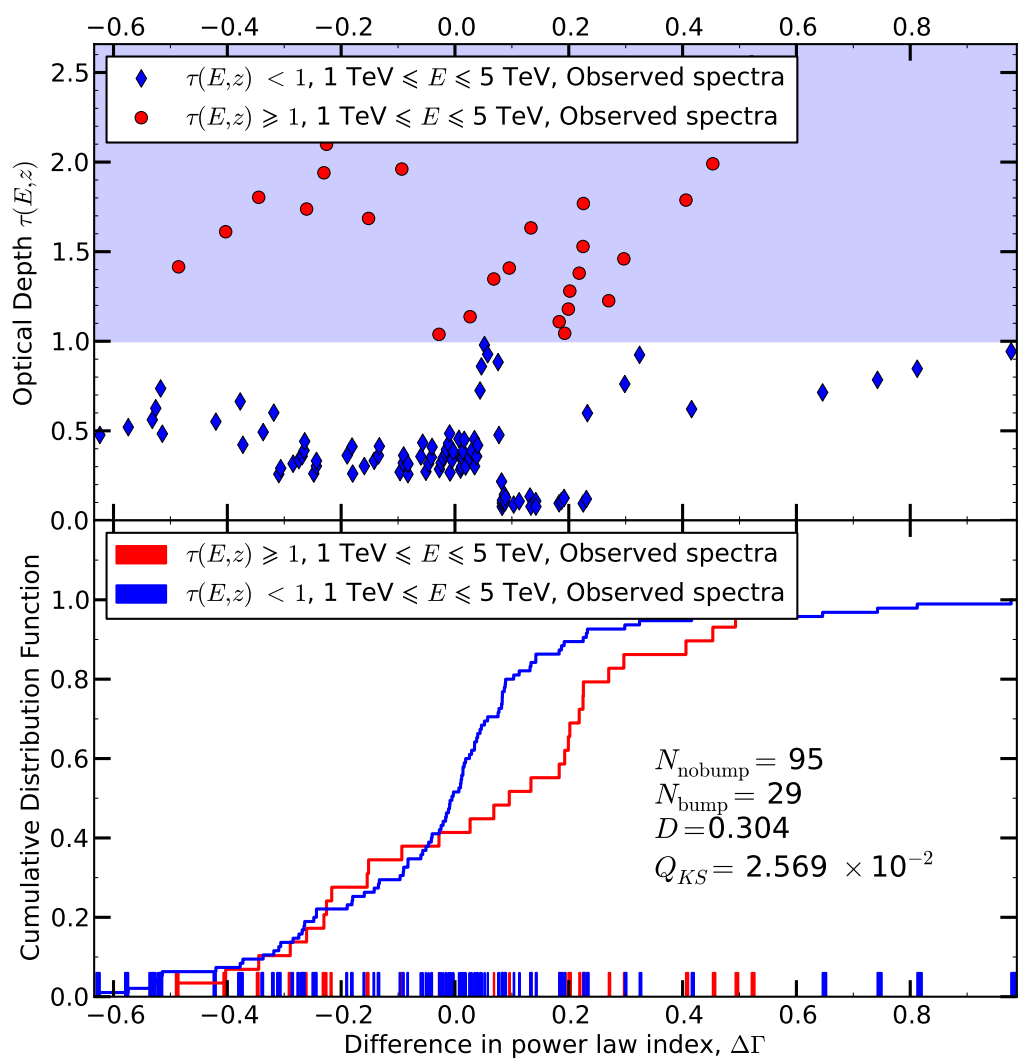

Figure 2: Results of the KS test searching for an EBL absorption feature in the observed spectra. The upper panel shows the optical depth against ratios of data points between $1 \mathrm{TeV} \leq E \leq 5 \mathrm{TeV}$. Ratios corresponding to $\tau_{\gamma} \geq 1$ (red circles) lie in the light-blue shaded region. In the lower panel the corresponding CDFs are shown. While the CDF of the ratios corresponding to $\tau_{\gamma}<1$ (blue line) increases symmetrically around $R=0$ the CDF for ratios with $\tau_{\gamma} \geq 1$ (red line) with a sharp increase around $R=0.2$.

mild significance which is reduced by deabsorbing the spectra as it is expected for a feature induced by the attenuation of cosmological $\gamma$-ray sources by the EBL.

\section{Conclusion}

In this article a new approach based on the Kolmogorov-Smirnov test is presented that allows to examine VHE spectra of AGN for deviations from standard physics and to search for an imprint of the EBL on these spectra. It includes every data point of every considered spectrum thus allowing for a large data sample to be analyzed. A further advantage of the approach is the fact that the observational errors only enter in the power law fits but not into the KS test itself. Hence, over- or underestimated errors do not affect the probabilities estimated here.

As a first result an evidence is found that the observed spectra deabsorbed by a lower limit EBL model cannot be described by power laws. This could imply that either the intrinsic AGN spectra cannot be described by power laws or that the universe is more transparent to VHE photons 


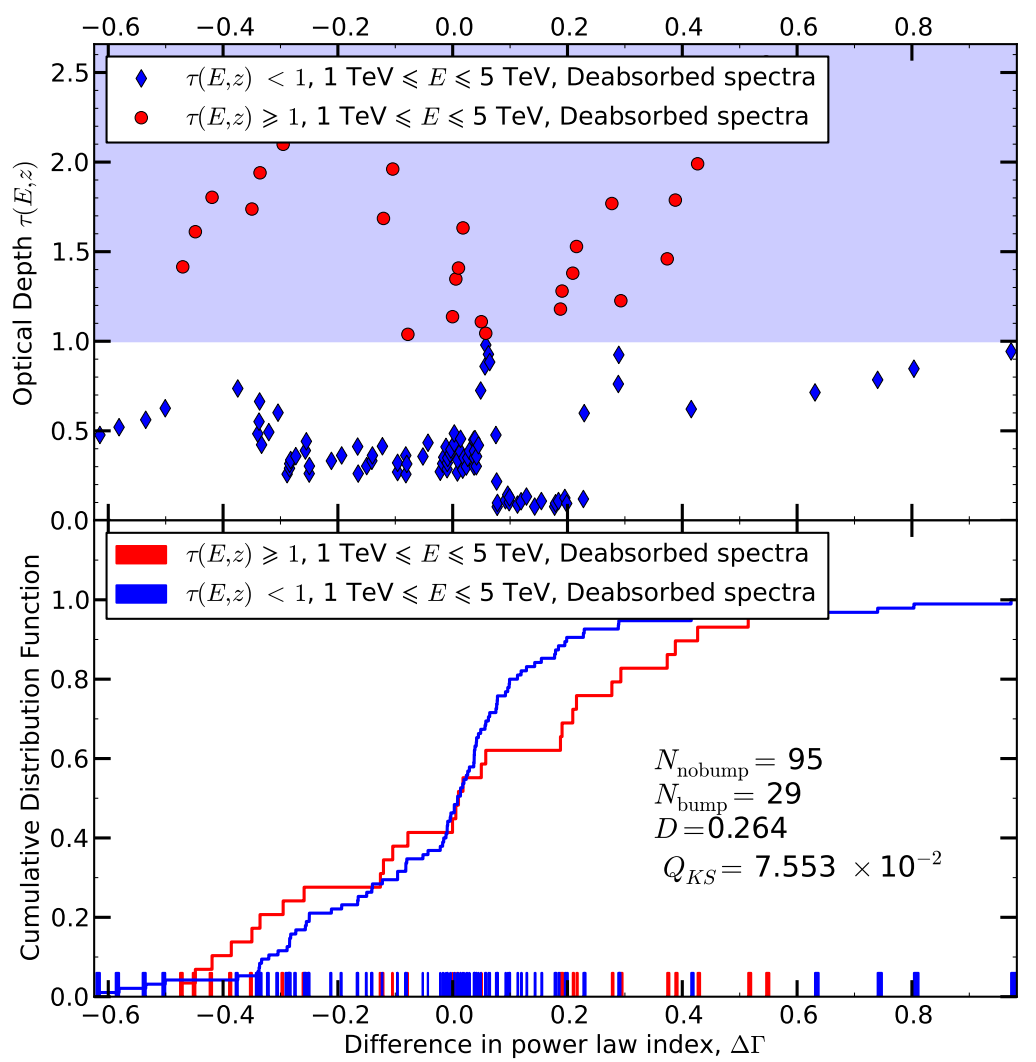

Figure 3: The same as Fig. 2 for the deabsorbed spectra. The ratios with $\tau_{\gamma} \geq 1$ are shifted towards $R=0$ which results in an increased significance that the two distributions are equal compared to the case of the observed spectra.

than predicted by a lower limit EBL model e.g. due to exotic mechanisms such as ALPs. For a definite answer more data has to be considered where in the near future $O(10)$ new AGN spectra should be published. ${ }^{3}$

Secondly, an absorption feature is found with a mild significance (the two distributions are different with a probability of $2.57 \times 10^{-2}$ ). The significance is reduced for the deabsorbed AGN spectra supporting the conclusion that this characteristic is indeed induced by the absorption of VHE photons by the EBL. This result has to be confirmed with more data and other EBL models [12] and it can be used to design a model which eliminates the absorption feature entirely, either by freely varying the entire EBL SED or by scaling the dust and starlight component relative to each other.

\section{Acknowledgements}

The authors would like to thank the organizers of the CRF 2010 workshop for the opportunity to present the results of this work on a poster. This work was made possible with the support of

\footnotetext{
${ }^{3}$ See e.g. http://tevcat.uchicago.edu/
} 
the LEXI young investigators group "Cosmic radiation fields and search for dark matter" at the university of Hamburg.

\section{References}

[1] R. J. Gould and G. P. Schréder, Pair Production in Photon-Photon Collisions, Physical Review 155 (Mar., 1967) 1404-1407.

[2] M. G. Hauser and E. Dwek, The Cosmic Infrared Background: Measurements and Implications, Ann. Rev. Astron. Astrophys. 39 (2001) 249-307, [arXiv : astro-ph/0105539].

[3] A. Franceschini, G. Rodighiero, and M. Vaccari, Extragalactic optical-infrared background radiation, its time evolution and the cosmic photon-photon opacity, Astron. Astrophys. 487 (Sept., 2008) 837-852, [0805. 1841].

[4] T. M. Kneiske and H. Dole, A lower-limit flux for the extragalactic background light, Astron. Astrophys. 515 (June, 2010) A19+, [1001.2132].

[5] A. Domínguez, J. R. Primack, D. J. Rosario, et al., Extragalactic background light inferred from AEGIS galaxy-SED-type fractions, Mon. Not. R. Astron. Soc. (Oct., 2010) 1665-+, [1007. 1459].

[6] U. Jacob and T. Piran, Inspecting absorption in the spectra of extra-galactic gamma-ray sources for insight into Lorentz invariance violation, Phys. Rev. D 78 (Dec., 2008) 124010-+, [0810 . 1318].

[7] L. Shao and B. Ma, Lorentz Violation Effects on Astrophysical Propagation of Very High Energy Photons, Modern Physics Letters A 25 (2010) 3251-3266, [1007. 2269].

[8] K. A. Hochmuth and G. Sigl, Effects of axion-photon mixing on gamma-ray spectra from magnetized astrophysical sources, Phys. Rev. D 76 (Dec., 2007) 123011-+, [0708. 1144].

[9] D. Hooper and P. D. Serpico, Detecting Axionlike Particles with Gamma Ray Telescopes, Physical Review Letters 99 (Dec., 2007) 231102-+, [0706.3203].

[10] A. Mirizzi and D. Montanino, Stochastic conversions of TeV photons into axion-like particles in extragalactic magnetic fields, JCAP 12 (Dec., 2009) 4-+, [0911.0015].

[11] M. A. Sánchez-Conde, D. Paneque, E. Bloom, F. Prada, and A. Domínguez, Hints of the existence of axionlike particles from the gamma-ray spectra of cosmological sources, Phys. Rev. D 79 (June, 2009) 123511-+, [0905.3270].

[12] M. Meyer and D. Horns, in preparation. 2011.

[13] A. Chen, L. C. Reyes, and S. Ritz, Detecting the Attenuation of Blazar Gamma-Ray Emission by Extragalactic Background Light with the Gamma-Ray Large Area Space Telescope, Astrophys. J. $\mathbf{6 0 8}$ (June, 2004) 686-691, [arXiv: astro-ph/0402152].

[14] M. Georganopoulos, J. D. Finke, and L. C. Reyes, A Method for Setting Upper Limits to the Extragalactic Background Light with Fermi-lat and TeV Observations of Blazars, Astrophys. J., Lett. 714 (May, 2010) L157-L161, [1004 .0017].

[15] W. H. Press, Numerical recipes in C++ : the art of scientific computing. 2002.

[16] A. Mirizzi, G. G. Raffelt, and P. D. Serpico, Signatures of axionlike particles in the spectra of TeV gamma-ray sources, Phys. Rev. D 76 (July, 2007) 023001-+, [0704 . 3044].

[17] F. Aharonian (for the HESS Collaboration) et al., Detection of VHE gamma-ray emission from the distant blazar 1ES 1101-232 with HESS and broadband characterisation, Astron. Astrophys. 470 (Aug., 2007) 475-489, [0705.2946]. 